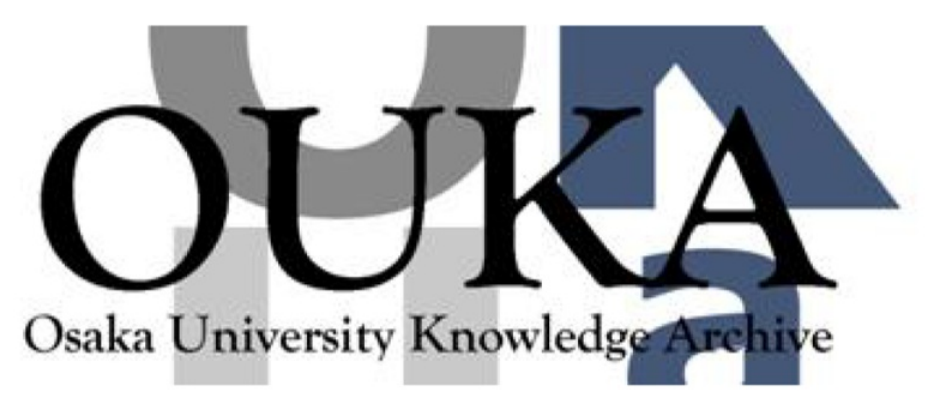

\begin{tabular}{|c|c|}
\hline Title & $\begin{array}{l}\text { Self-limiting oxidation of SiGe alloy on } \\
\text { silicon-on-insulator wafers }\end{array}$ \\
\hline Author(s) & $\begin{array}{l}\text { Shimura, Takayoshi; Shimizu, Michihiro; } \\
\text { Horiuchi, Shinichiro et al. }\end{array}$ \\
\hline Citation & Applied Physics Letters. 89(11) p. 111923 \\
\hline Issue Date & $2006-09-11$ \\
\hline oaire:version & VoR \\
\hline URL & https://hdl. handle. net/11094/85481 \\
\hline rights & $\begin{array}{l}\text { This article may be downloaded for personal use } \\
\text { only. Any other use requires prior permission } \\
\text { of the author and AIP Publishing. This article } \\
\text { appeared in Appl. Phys. Lett. and may be found } \\
\text { at https://doi.org/10.1063/1.2353812. }\end{array}$ \\
\hline Note & \\
\hline
\end{tabular}

Osaka University Knowledge Archive : OUKA

https://ir. Library. osaka-u. ac. jp/

Osaka University 


\section{Self-limiting oxidation of SiGe alloy on silicon-on-insulator wafers}

Cite as: Appl. Phys. Lett. 89, 111923 (2006); https://doi.org/10.1063/1.2353812

Submitted: 12 May 2006 • Accepted: 20 July 2006 • Published Online: 15 September 2006

Takayoshi Shimura, Michihiro Shimizu, Shinichiro Horiuchi, et al.

View Online

\section{ARTICLES YOU MAY BE INTERESTED IN}

Oxidation studies of SiGe

Journal of Applied Physics 65, 1724 (1989); https://doi.org/10.1063/1.342945

Effects of Ge concentration on SiGe oxidation behavior

Applied Physics Letters 59, 1200 (1991); https://doi.org/10.1063/1.105502

Kinetics and mechanism of oxidation of SiGe: dry versus wet oxidation

Applied Physics Letters 54, 644 (1989); https://doi.org/10.1063/1.100905

母QBLOX

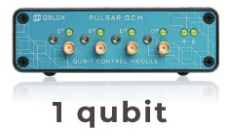

Shorten Setup Time Auto-Calibration More Qubits

Fully-integrated Quantum Control Stacks Ultrastable DC to $18.5 \mathrm{GHz}$ Synchronized $<<1$ ns Ultralow noise

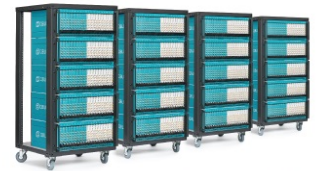

100s qubits

visit our website > 


\title{
Self-limiting oxidation of SiGe alloy on silicon-on-insulator wafers
}

\author{
Takayoshi Shimura ${ }^{a}$ \\ Department of Precision Science and Technology, Graduate School of Engineering, Osaka University, \\ 2-1 Yamadaoka, Suita, Osaka 565-0871, Japan and Venture Business Laboratory, Center for Advanced \\ Science and Innovation, Osaka University, 2-1 Yamadaoka, Suita, Osaka 565-0871, Japan \\ Michihiro Shimizu, Shinichiro Horiuchi, Heiji Watanabe, and Kiyoshi Yasutake \\ Department of Precision Science and Technology, Graduate School of Engineering, Osaka University, \\ 2-1 Yamadaoka, Suita, Osaka 565-0871, Japan \\ Masataka Umeno \\ Department of Management and Information Science, Faculty of Engineering, \\ Fukui University of Technology, 3-6-1 Gakuen, Fukui, Fukui 910-8585, Japan
}

(Received 12 May 2006; accepted 20 July 2006; published online 15 September 2006)

\begin{abstract}
Self-limiting oxidation of SiGe alloy on silicon-on-insulator wafers was investigated. For oxidation at $1000{ }^{\circ} \mathrm{C}$, oxidation stops completely after a few hours for the $\mathrm{Si}_{1-x} \mathrm{Ge}_{x}(x=0.068-0.16)$ layers . For higher initial Ge concentrations of the $\mathrm{SiGe}$ layer, the oxidation saturated in a shorter oxidation time, whereas saturation was not observed for the oxidation at 900 and $1100{ }^{\circ} \mathrm{C}$. The authors propose a model for self-limiting oxidation, in which the oxidation saturation is governed by an interfacial Ge-rich layer that depends on the oxidation temperature and the initial $\mathrm{Ge}$ concentration. (C) 2006 American Institute of Physics. [DOI: 10.1063/1.2353812]
\end{abstract}

Recently, oxidation of SiGe alloy has been employed in the fabrication of SiGe-on-insulator (SGOI) substrates. ${ }^{1,2}$ A strained Si layer is epitaxially grown on a relaxed SiGe surface, and it is used as the channel for metal insulator semiconductor field effect transistors (MISFETs). Devices which have a strained Si channel are expected to be used as highspeed MISFETs, because carrier mobility in strained $\mathrm{Si}$ is higher than that in relaxed $\mathrm{Si}^{3,4}$

In the oxidation process of $\mathrm{SiGe}$ alloy $\mathrm{Ge}$ atoms are ejected from the interface between the surface oxide and the $\mathrm{SiGe}$ layers into the $\mathrm{SiGe}$ substrate. During the oxidation of the SiGe layer on the silicon-on-insulator (SOI) substrate, Ge atoms accumulate at the $\mathrm{SiO}_{2} / \mathrm{SiGe}$ interface and diffuse towards the bottom of the SiGe layer. The concentration of $\mathrm{Ge}$ in the layers between the top oxide layer and the buried oxide (BOX) layer increases on further oxidation. It is considered that this Ge-condensation method produces a relaxed and thin SiGe layer with a high Ge fraction and low dislocation density, because the Ge concentration in the initial SiGe layer is sufficiently low to suppress dislocation and the slippage at the BOX layer interface occurs during condensation. ${ }^{1}$

It is, therefore, important to understand the oxidation mechanism of SiGe alloy. A considerable number of studies have reported the enhancement of the oxidation rate of SiGe alloy compared with that for pure $\mathrm{Si}^{5}{ }^{5}$ However, there have been very few studies done on the reduction in the oxidation rate, as observed by Eugéne et al. ${ }^{6}$ and Tezuka et al. ${ }^{1}$ In this present study we investigated this reduction in the oxidation rate and observed self-limiting oxidation of SiGe alloy on a SOI wafer. We present the experimental evidence for this self-limiting oxidation of SiGe alloy and discuss the mechanism for this phenomenon.

Bonded SOI wafers with a $60 \mathrm{~nm}$ top Si layer and a $140 \mathrm{~nm}$ BOX layer were used as the starting substrate. The

${ }^{a)}$ Electronic mail: shimura@prec.eng.osaka-u.ac.jp
$\mathrm{Si}_{1-x} \mathrm{Ge}_{x}$ layers $(x=0-0.16)$ with an average thickness of $188 \mathrm{~nm}$ were epitaxially grown on the SOI substrate in a molecular-beam-epitaxy chamber with an electron gun for evaporating $\mathrm{Si}$ and a Knudsen cell for evaporating Ge. The substrates were maintained at $480{ }^{\circ} \mathrm{C}$ during growth. Condensation of $\mathrm{Ge}$ was carried out by repeating oxidation at $900-1100{ }^{\circ} \mathrm{C}$ for $1 \mathrm{~h}$ in a $100 \%$ dry- $\mathrm{O}_{2}$ atmosphere. The thickness of the oxide layer and the Ge concentration of the SiGe layer were measured by spectroscopic ellipsometry after each oxidation cycle. In order to obtain the depth profile of the Ge concentration, x-ray photoelectron spectroscopy (XPS) was employed using $\mathrm{Ar}^{+}$sputter etching after removing the surface oxide layer by HF solution. The lattice strain of the SiGe layer was evaluated by $\mathrm{x}$-ray reciprocal-space maps obtained using a rotating anode $\mathrm{x}$-ray generator.

Figure 1 shows the oxide thickness on the $\mathrm{SiGe}$ layer as a function of the oxidation time. The thickness of the oxide layer increases with the oxidation time for the $\mathrm{Si}_{1-x} \mathrm{Ge}_{x}(x$ $=0-0.054)$ layers. We see that there is a tendency for the

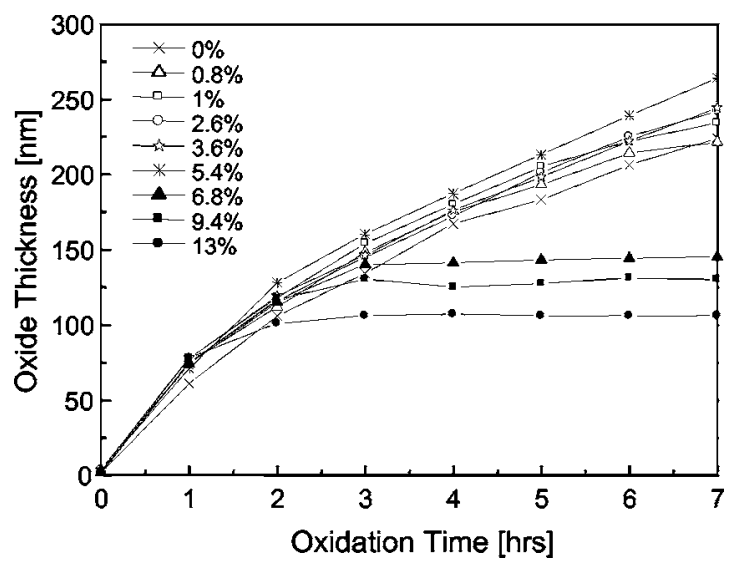

FIG. 1. Oxide thickness as a function of oxidation time. Ge concentrations in the initial $\mathrm{SiGe}$ alloy ranged from $0 \%$ to $13 \%$. Oxidation temperature is $1000{ }^{\circ} \mathrm{C}$. 


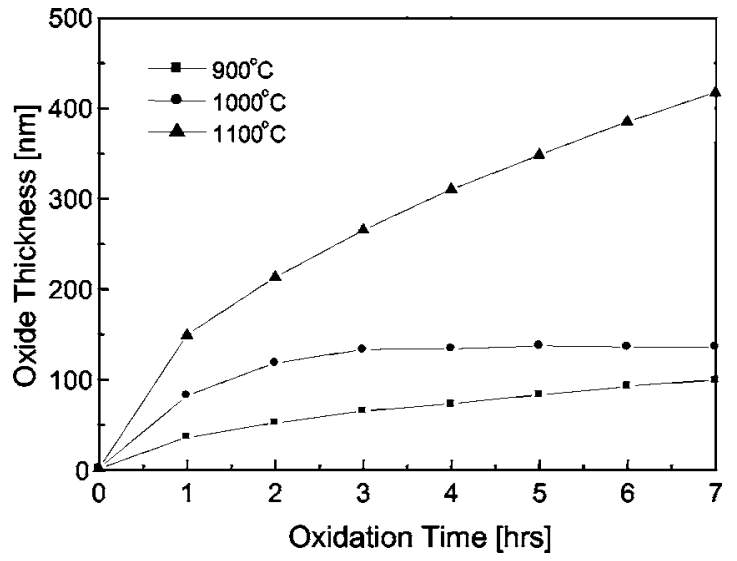

FIG. 2. Oxide thickness as a function of oxidation time. Ge concentration in the initial $\mathrm{SiGe}$ alloy is $12 \%$. Oxidation temperatures ranged from 900 to $1000{ }^{\circ} \mathrm{C}$.

oxidation rate to be higher for higher Ge concentrations in the initial SiGe alloy. This enhancement of the SiGe oxidation rate is in agreement with previous studies. ${ }^{5}$ The oxidation rate does not increase with the oxidation time, although the Ge concentration of the SiGe layer increases. This is because the oxidation rate is limited by the diffusion of oxygen through the thick surface oxide layers. A characteristic feature in this figure is that oxidation stopped completely in the $\mathrm{Si}_{1-x} \mathrm{Ge}_{x}(x=0.068-0.13)$ layers after a few hours. For higher Ge concentrations, oxidation saturates after a shorter time, resulting in a thinner oxide layer.

In Fig. 2 the effect of the oxidation temperature on the oxidation rate is shown for the SiGe alloy having an initial Ge concentration of $12 \%$. As the oxidation temperature increases, the oxidation rate increases during the initial stage of oxidation. Saturation of oxidation occurs for the sample oxidized at $1000{ }^{\circ} \mathrm{C}$ as Fig. 1 shows. Note that the thickness of the oxide layer for the sample oxidized at $1100{ }^{\circ} \mathrm{C}$ continues to increase after $7 \mathrm{~h}$, even though its thickness is much greater than the saturation thickness of the sample oxidized at $1000{ }^{\circ} \mathrm{C}$. In addition, oxidation saturation was not observed for the sample oxidized at $900{ }^{\circ} \mathrm{C}$ even after $7 \mathrm{~h}$.

In order to clarify the mechanism for this self-limiting oxidation it is important to investigate the depth profile of the Ge concentration. Figure 3 shows the depth profiles of the Ge concentrations in the SiGe layers oxidized for $0,1,4$, and $7 \mathrm{~h}$. The depth is estimated by converting the etching time of $\mathrm{Ar}^{+}$sputter into depth on the basis of the thickness of the SiGe layers measured by ellipsometry; the position of the BOX layer is estimated by the depth profile of the $\mathrm{O}$ concentration. Figure 3(a) shows the results for a sample having an initial Ge concentration of $5.1 \%$. We see that Ge atoms accumulate under the oxidized interface and diffuse into the SOI layer with increasing oxidation time. The total amount of Ge atoms remains constant within the accuracy of the measurement, indicating that the Ge atoms condense into the $\mathrm{SiGe}$ layer without $\mathrm{Ge}$ atoms being incorporated into the oxide layer.

In Fig. 3(b) the Ge concentration depth profiles are shown for the samples oxidized for $0-7 \mathrm{~h}$ having an initial Ge concentration of $16 \%$. It is surprising that the profile for the sample oxidized for $7 \mathrm{~h}$ is almost the same as that for the sample oxidized for $4 \mathrm{~h}$, even though it was oxidized at $1000{ }^{\circ} \mathrm{C}$ for an additional $3 \mathrm{~h}$. A characteristic feature is the
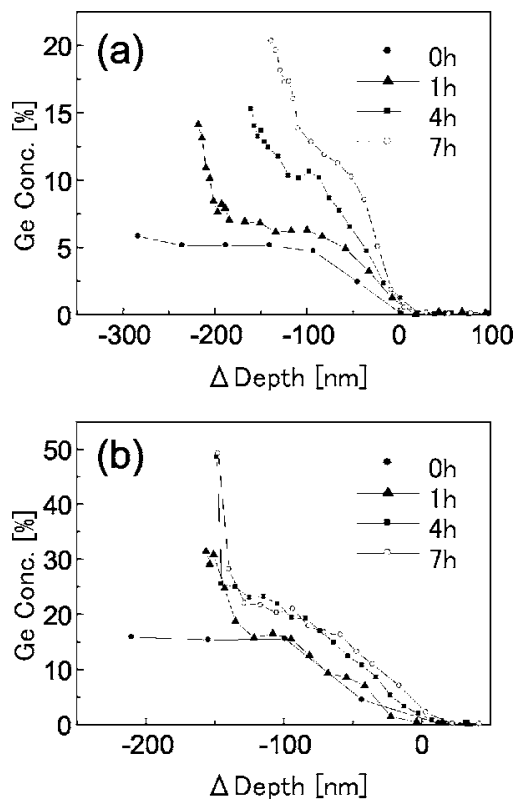

FIG. 3. Depth profiles of Ge concentration in SiGe layers oxidized for 0,1 , 4 , and $7 \mathrm{~h}$. The initial concentrations of $\mathrm{Ge}$ in the SiGe layers are $5.1 \%$ for (a) and $16 \%$ for (b). The interface between the SiGe and BOX layers is located at $\Delta$ Depth $=0 \mathrm{~nm}$.

abrupt increase below the top interface of the SiGe layers. It was also found by angle-resolved XPS measurement that the Ge concentration of the interfacial layer is $80 \%$ or greater, indicating the existence of a very thin layer, a few nanometers thick, with a Ge concentration of $80 \%$ or higher. These results indicate that the diffusion of $\mathrm{Ge}$ atoms just below the oxide layer is suppressed as well as the oxidation reaction of the SiGe alloy being suppressed.

X-ray reciprocal-space maps around the 113 Bragg point were obtained from the $\mathrm{Si}_{1-x} \mathrm{Ge}_{x}(x=0.16)$ samples. The reflection of the as-grown sample is observed close to the line of $H, K=1.00$, indicating that the SiGe layer is pseudomorphic. However, the position of the reflection of the SiGe layer oxidized for $4 \mathrm{~h}$ indicates that the strain in the $\mathrm{SiGe}$ layer is almost relaxed. The peak of the SiGe layer is weak and broad, showing that the crystalline quality of this layer is poor.

The diffusion coefficient of $\mathrm{Ge}$ in $\mathrm{SiGe}$ alloy depends on the Ge concentration. The coefficients at $1000{ }^{\circ} \mathrm{C}$ were reported to be $1.08 \times 10^{-16}-1.03 \times 10^{-15}$ in $\mathrm{Si}$ and 1.21 $\times 10^{-13} \mathrm{~cm}^{2} / \mathrm{s}$ in $\mathrm{Si}_{0.776} \mathrm{Ge}_{0.224}{ }^{7-10}$ The respective diffusion lengths are 6-19 and $209 \mathrm{~nm} / \mathrm{h}$. It is clear that the suppres-

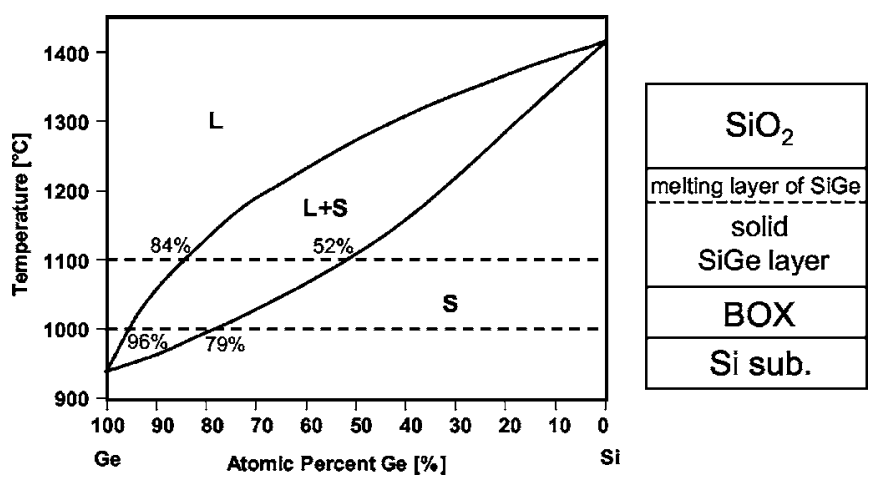

FIG. 4. Phase diagram of Si-Ge system (Ref. 11) and a schematic drawing of the melting layer for the saturated SiGe layer. 
sion of the diffusion of $\mathrm{Ge}$ atoms just below the surface oxide layer cannot be explained by a diffusion mechanism based on the concentration gradient.

Tezuka et al. proposed that the strain in the SiGe layer is one possible reason for the reduction in the oxidation rate of the SiGe layer on the basis of measurements of the residual strain in SGOI layers by Raman spectroscopy. ${ }^{1}$ The strain in $\mathrm{SiGe}$, arising from the gradient of the Ge fraction, suppresses the diffusion of $\mathrm{Si}$ to the oxidized interface through the $\mathrm{SiGe}$ layer. However, we found that the strain in the SiGe layer oxidized for $4 \mathrm{~h}$ was almost relaxed for the $\mathrm{Si}_{1-x} \mathrm{Ge}_{x}(x$ $=0.16)$ sample using $x$-ray reciprocal-space map technique, indicating that the oxidation stops for the sample where there is no strain in the SiGe layer. This means that the selflimiting oxidation is not due to lattice strain in the SiGe layer.

Instead, we propose a model in which the self-limiting oxidation is due to the melting of an interfacial layer having a high Ge concentration between the surface oxide and the SiGe layers (Fig. 4). As the oxidation time increases, Ge concentration just below the surface oxide layer increases. According to the phase diagram for the $\mathrm{Si}-\mathrm{Ge}$ system, ${ }^{11}$ the interfacial layer melts when the concentration becomes more than $79 \%$ for the oxidation at $1000{ }^{\circ} \mathrm{C}$. The interfacial layer consists of a mixture of liquid and solid phases having $\mathrm{Ge}$ concentrations of $96 \%$ and $79 \%$, respectively. If it is assumed that the mixed layer separates into liquid and solid layers to reduce the interfacial free energy between the liquid and solid phases and that the liquid layer is adjacent to the oxidized interface, then the oxidation rate would rapidly decrease, since the Si concentration is just $4 \%$ at the oxidized interface. The diffusion of Ge atoms would also be suppressed because the liquid and solid layers are stable at $1000{ }^{\circ} \mathrm{C}$.

Saturation of oxidation is not observed for the sample oxidized at $1100{ }^{\circ} \mathrm{C}$, even though the interfacial layer melts. In this case the $\mathrm{Si}$ concentration of the liquid layer covering the oxidized interface is $16 \%$, which is four times higher than that for the oxidation carried out at $1000{ }^{\circ} \mathrm{C}$. This is the reason why oxidation is not saturated at $1100{ }^{\circ} \mathrm{C}$. Saturation is not expected for oxidation at $900{ }^{\circ} \mathrm{C}$, because this temperature is below the melting point of pure Ge.

In conclusion, we have experimentally demonstrated the phenomenon of self-limiting oxidation of SiGe alloy. For oxidation at $1000{ }^{\circ} \mathrm{C}$, oxidation stopped completely after a few hours of oxidation of the $\mathrm{Si}_{1-x} \mathrm{Ge}_{x}(x=0.068-0.16)$ layers. For higher initial concentrations of $\mathrm{Ge}$ in the SiGe layer, the oxidation saturated in a shorter time. Saturation was not observed for the oxidation at 900 and $1100{ }^{\circ} \mathrm{C}$. To explain these results we propose a model in which the self-limiting oxidation is caused by melting of an interfacial layer having a high Ge concentration.

This work was partially supported by a Grant-in-Aid for Scientific Research and 21st Century COE Research, Center for Atomistic Fabrication Technology, from the Ministry of Education, Culture, Sports, Science and Technology.

${ }^{1}$ T. Tezuka, N. Sugiyama, T. Mizuno, M. Suzuki, and S. Takagi, Jpn. J. Appl. Phys., Part 1 40, 2866 (2001).

${ }^{2}$ T. Tezuka, N. Sugiyama, and S. Takagi, Appl. Phys. Lett. 79, 1798 (2001).

${ }^{3}$ T. Mizuno, S. Takagi, N. Sugiyama, J. Koga, T. Tezuka, K. Usuda, T. Hatakeyama, A. Kurobe, and A. Toriumi, Tech. Dig. - Int. Electron Devices Meet. 1999, 934.

${ }^{4}$ T. Mizuno, S. Takagi, N. Sugiyama, H. Satake, A. Kurobe, and A. Toriumi, IEEE Electron Device Lett. 21, 230 (2000).

${ }^{5}$ S. C. Jain and P. Balk, Thin Solid Films 223, 348 (1993) and references therein.

${ }^{6}$ J. Eugéne, F. K. LeGoues, V. P. Kesan, S. S. Lyer, and F. M. d'Heurle, Appl. Phys. Lett. 59, 78 (1991).

${ }^{7}$ M. Ogino, Y. Oana, and M. Watanabe, Phys. Status Solidi A 72, 535 (1982).

${ }^{8}$ P. Dorner, W. Gust, B. Predel, U. Roll, A. Lodding, and H. Odelius, Philos. Mag. A 49, 557 (1984).

${ }^{9}$ N. Sugii, J. Appl. Phys. 89, 6459 (2001).

${ }^{10}$ G. L. McVay and A. R. DuCharme, Phys. Rev. B 9, 627 (1974).

${ }^{11}$ Binary Alloy Phase Diagrams (American Society for Metals, Metals Park, OH, 1986), Vol. 2, p. 1248. 